\title{
Management of Acute Conjunctivitis
}

\author{
Kira L. Segal · Edward C. Lai - Christopher E. Starr
}

Published online: 26 July 2014

(C) Springer Science + Business Media New York 2014

\begin{abstract}
Acute conjunctivitis can be caused by viral, bacterial, and allergic sources. There is growing need for rapid diagnostics and effective therapies given the significant economic burden and pervasive nature of the disease. Additionally, with growing microbial resistance, practice guidelines should encourage appropriate use of antibiotics, limited to clinical situations with proven benefit. New inoffice detectors may enable rapid definitive diagnosis among clinically similar entities. Development of and studies examining steroid-sparing immunomodulators for conjunctivitis are warranted.
\end{abstract}

Keywords Conjunctivitis - Acute conjunctivitis · Viral conjunctivitis - Bacterial conjunctivitis - Allergic conjunctivitis · Adenovirus

\section{Introduction}

Acute conjunctivitis is a common problem caused by both infectious and noninfectious sources. As the most prevalent ocular condition encountered in the primary care setting, acute conjunctivitis accounts for up to $2 \%$ of outpatient primary care and $1 \%$ of emergency room visits $[1,2]$. Only $20 \%$ of cases initially present to an optometrist or

\section{K. L. Segal · E. C. Lai · C. E. Starr $(\bowtie)$}

Department of Ophthalmology, Weill Cornell Medical College,

NewYork-Presbyterian Hospital, 1305 York Avenue, 11th and

12th Floors, New York, NY 10065, USA

e-mail: cestarr@med.cornell.edu

K. L. Segal

e-mail: kls2003@nyp.org

E. C. Lai

e-mail: ec12001@med.cornell.edu ophthalmologist; in the majority of cases, they first present to a primary care provider [2]. The economic burden of conjunctivitis is significant. For bacterial conjunctivitis alone, estimates for direct and indirect costs approach $\$ 857$ million annually in the USA [3]. Allergic conjunctivitis is by far the most frequent subtype, affecting $15-40 \%$ of the population [4]. Treatment of acute conjunctivitis is determined primarily by the underlying etiology, despite the challenge of making a definitive diagnosis. Due to symptom overlap and varied clinical presentation, studies demonstrate clinical inaccuracy to range from 40-75\% [5-9]. Nevertheless, ophthalmologists still treat conjunctivitis based on clinical suspicion supplemented by epidemiological data and available laboratory testing.

\section{Viral Conjunctivitis}

Of all infectious etiologies, viral conjunctivitis is the most common, accounting for up to $80 \%$ of all cases $[5,10,11]$. Adenovirus causes between $65-90 \%$ of such cases [12-14], and manifests as three distinct clinical syndromes. Diagnosis of viral conjunctivitis can be aided by clinical history, i.e., the presence of comorbid upper respiratory tract infection, cold sores/rash, exposure to children or sick contacts, and prior history of conjunctivitis in the fellow eye. Important ocular symptoms to elicit include timing of symptom onset, presence of itching, type of discharge, matting of eyelids, pain, photophobia, and vision change [15]. Slit lamp findings more consistent with viral etiologies include serous or serosanguineous discharge, pseudomembranes, chemosis, follicular reaction, large subconjunctival hemorrhage and micro-hemorrhages, and corneal subepithelial infiltrates [5, $6,10,16-18]$. Tender preauricular lymphadenopathy can be found in up to $50 \%$ of patients [16]. 
Duration of incubation and infectivity for adenoviralassociated conjunctivitis is estimated to be 5-12 and 10-14 days, respectively [9]. However, studies demonstrate that active virus can linger on environmental surfaces for up to 5 weeks $[19,20]$. The high transmission rate to family members and close contacts of the patient $(10-50 \%)$ render viral precautions essential $[9,21]$. Our recommendation is to remain out of work for the duration of symptoms, specifically as long as eyes are red and tearing. Additionally, as is standard practice, close attention to hand washing and limiting hand contact is emphasized to every patient.

Treatment of viral conjunctivitis is controversial. Topical antibiotics are not indicated for confirmed viral infection, particularly given rising antimicrobial resistance. Further, with topical medications, patients may spread involvement to the healthy eye through contact with the topical medication bottle. In general, viral conjunctivitis is treated with supportive care including cold compresses, refrigerated artificial tears, and topical antihistamines.

Steroids are typically withheld in the acute phase unless there is substantial corneal, subepithelial infiltrates (SEIs) with reduced vision, and/or conjunctival inflammation (membranes). A review by Everitt et al. [22] comparing steroid and antiviral therapy concluded there was limited benefit to treatment although the studies were underpowered and of poor quality. In a randomized placebo-controlled trial of topical steroids, 111 patients presenting with follicular conjunctivitis were randomized to receive either dexamethasone $0.1 \%$ or hypromellose $0.3 \%$ drops four times daily for 1 week. Patients were then examined 5-7 days after presentation and asked for subjective responses regarding symptoms. Although the study was limited by patient recruitment, the patients receiving topical steroid were more likely to report improvement from the drop. There was no significant difference in graders' rating of hyperemia. Patients in the placebo group were more likely to later develop subepithelial infiltrates, but this value did not reach significance. None of the patients developed signs of HSV despite topical steroid use [23••]. In three earlier studies of topical steroid use for viral conjunctivitis, topical steroids were not effective in hastening recovery. Specifically, in 53 patients with culture proven adenovirus type 8 , steroid-neomycin drops (vs. lubricants) did not change the course of the conjunctivitis or decrease risk of progression to nummular or punctate keratitis [24]. In a randomized study of 74 US military personal, dexamethasone (vs. trifluridine or artificial tears) did not reduce patient symptoms or time confined to barracks [25•]. In a randomized study of prednisolone acetate and phenylephrine hydrochloride drops compared to placebo, more patients in the placebo group developed SEIs, and this difference did reach significance [26••]. This was the only outcome measured in the study.

Use of steroid in acute stages of infection should be used judiciously. Over the short term, use of steroid can prolong viral replication time as demonstrated with $1 \%$ prednisolone acetate in a rabbit model [27•-0]. Further, there is a theoretical risk to uncover underlying HSV in presumed adenoviral conjunctivitis, though this has not been demonstrated in the literature. In a study by Uchio et al. [28••], $4.8 \%(n=23)$ of samples taken from patients with clinical symptoms consistent with epidemic keratoconjunctivitis (EKC) were positive for HSV-1. Nine of these patients were treated with steroids for superficial punctate keratitis (SPK) or diffuse SEIs and none developed worsened herpetic corneal manifestations.

Subepithelial infiltrates, which reflect a delayed inflammatory reaction, can develop 1-2 weeks following infection with adenovirus. By confocal microscopy, SEIs are dendritic cells arranged in intricate networks overlying hyperreflective cellular plaques in the anterior stroma [29]. Infiltrates may disappear without long-term sequelae or the lesions may remain in the cornea chronically causing vision loss, photophobia, astigmatism, halo, and glare. Various therapies are considered for treatment of subepithelial infiltrates. Topical corticosteroids are commonly utilized, though there is a risk of recurrence of infiltrates following corticosteroid withdrawal and long-term steroid use can be hampered by side effects such as elevated intraocular pressure, cataract formation, and infection [26••]. Topical cyclosporine is effective for treatment of SEIs in concentrations of $0.05 \%$ (Restasis $^{\mathrm{TM}}$, Allergan, Irvine, California, USA), $1 \%$, and $2 \%$. Success rates for eliminating SEIs with topical cyclosporine range from $66-100 \%$, though many patients have difficulty tapering off treatment [30-33, 34*0]. Like corticosteroids, topical cyclosporine at all doses may increase the viral replication potential and prolong the duration of viral shedding [34 ••]. Photorefractive keratectomy (PRK) and phototherapeutic keratectomy (PTK) with mitomycin $\mathrm{C}$ are emerging as alternative surgical therapies. Yamazaki et al. [35॰] performed PTK to eliminate SEIs following EKC in 28 of 31 eyes. PRK offers the added benefit of refractive correction in myopic patients although corneal stability may be an issue [36.]. One case report demonstrated ablation of SEIs in the central but not peripheral cornea following PRK [37]. Although promising, larger, randomized controlled trials are needed to examine these yet unproven treatment strategies.

There is growing laboratory and clinical evidence for the use of topical antivirals for the treatment of adenovirusassociated conjunctivitis. In two single case reports, topical cidofovir led to successful resolution of symptoms [38, 39]. In a larger study, it was shown to reduce corneal opacities, though these results were accompanied by side effects [40]. 
In other studies, cidofovir \pm cyclosporine did not alter the duration of symptoms [31]. Comparing cidofovir to placebo in animal models consistently demonstrated reduction of viral titers and duration of shedding of adenovirus (AD5 strain) [41-43, 44•]. Toxicity including redness of eyelids, conjunctival injection, and narrowing of the lacrimal canaliculus were seen $[45,46]$. Polyvinylpyrrolidone-iodine (Betaisodona) and povidone-iodine and topical dexamethasone drops $(n=6)$ both demonstrated modest improvement in symptoms, though the former did not decrease development of corneal infiltrates [47, 48]. A new formulation of $0.1 \%$ /povidone-iodine $0.4 \%$ (FST-100, Foresight Biotherapeutics) reduced clinical symptoms and viral titers with limited toxicity in preclinical studies [49••]. Clinical trials with this compound are ongoing. Topical ganciclovir $(3,1$, or $0.3 \%)$ tested in cotton rat eyes demonstrated a trend toward reduced incidence, duration, and titers of virus shed in eyes treated with the $3 \%$ concentration. The values did not reach significance [50]. Ganciclovir $0.15 \%$ ophthalmic gel compared to preservative free artificial tears also showed a trend toward earlier clinical improvement, lower transmission rates to the fellow eye and close contacts, but did not reach statistical significance [51••]. Clinical trials are currently underway comparing topical ganciclovir (Zirgan, Bausch+Lomb) to genteal gel for treatment of adenoviral conjunctivitis.

A rapid point of care (POC) immunoassay, the RPS Adeno Detector (RPS AD1, a product of Rapid Pathogen Screening, Inc.) has emerged as a clinical tool to identify adenovirus as the source of conjunctivitis in the office setting. When compared to the gold standard confirmatory immunofluorescence (CC-IFA), which takes 7 days to result, the RPS adeno detector offers $88 \%$ sensitivity and $91 \%$ specificity [52] with results in $10 \mathrm{~min}$. Recently, a second-generation device, the AdenoPlus (RPS ADP; Rapid Pathogen Screening Inc.), was FDA approved with the added benefit of detection of all 55 adenoviral serotypes [53・•]. Like the rapid strep test commonly used in pediatric practices, rapid adenoviral testing may help to distinguish between the various forms of acute conjunctivitis and subsequently reduce the use of unnecessary antibiotics. Additionally, a repeat negative test of a previously positive patient suggests decreased viral burden and may expedite return to work or school. One economic analysis estimated that rapid point of care testing could save an estimated $\$ 429.4$ million per year in the USA and avoid 1.1 million cases of unnecessary antibiotic use [2].

\section{Herpes Conjunctivitis}

Herpes simplex (HSV) virus is ubiquitous and its associated conjunctivitis is often difficult to distinguish from adenovirus-related conjunctivitis and may be present in $1-5 \%$ of all conjunctivitis cases [52]. Clinical diagnosis becomes challenging without the presence of skin lesions, highlighting the importance of a thorough history, which may elicit prior evidence of suggestive cold sores. Unilateral presentation is more common and discharge is thin and watery. Blepharoconjunctivitis is the most common single form of HSV in children, accounting for $41.5 \%$ of HSV-infected patients in one study [54]. The goal of treating HSV-associated conjunctivitis is to prevent corneal infection. Topical or oral treatments are recommended. Ganciclovir gel $0.15 \% 3-5$ times per day and trifluridine $1 \%$ 5-9 times per day are topical options; ganciclovir is preferred due to less corneal toxicity as that seen with trifluridine use $[55,56 \bullet \bullet, 57,58]$. Oral acyclovir 200-400 mg, 5 times per day is most commonly prescribed, however, valacyclovir or famciclovir can also be used. Oral antiviral agents seem to be well tolerated in children, although dose adjustment is required, and are recommended for prophylaxis of keratitis in children with recurrent blepharoconjunctivitis [59]. Steroids should be avoided if HSV is suspected given the potential to worsen infection.

Varicella (Herpes) Zoster Virus (HZV)-associated conjunctivitis is second only to the eyelid as the most common site of ocular involvement in herpes zoster ophthalmicus [60]. Particular attention should be paid to the ophthalmologic exam with the presence of vesicles on the tip of the nose (Hutchinson sign). Patients may present with painful eyelid ulceration and conjunctival vesicles. Rupture of eyelid vesicles can lead to secondary bacterial infection and subsequent cicatricial changes of the conjunctiva. Topical antibiotic ointments can be used to prevent secondary infection and provide symptomatic relief from vesicle rupture. Additionally, the presence of HZV conjunctivitis signals potential for involvement of other ocular structures. Our practice is to use oral antivirals such as acyclovir (800 mg five times daily for 7-10 days), valacyclovir, or famciclovir in additional to antibiotic ointment to the skin areas [61]. Treatment of concurrent ocular manifestations is warranted pending the specific clinical scenario.

\section{Bacterial Conjunctivitis}

Bacterial conjunctivitis is the second most common infectious etiology of conjunctivitis in adults, and it is the most common infectious etiology identified in pediatric patients. Clinical presentation may be nonspecific and bacterial conjunctivitis can be confused with viral and even noninfectious sources. In a prospective study by Rietveld et al. [62] bilateral matting of the eyelids, lack of itching, and no history of conjunctivitis most strongly predicted a 
bacterial etiology. Mucopurulent discharge is classic for bacterial etiology.

Mild bacterial conjunctivitis is usually self-limited in otherwise healthy adults and it is reasonable to simply observe these patients. If used in the first $2-5$ days, topical antibiotics can hasten resolution of symptoms in culture positive disease [63]. If administered after 5 days of initial symptoms, effect of treatment will be minimal. In adults, the most common pathogens identified are Staphylococcus, followed by Streptococcus pneumonia and Haemophilus. No antibiotic in the literature has been identified as superior to any other, although based on microbial patterns, a broad-spectrum antibiotic is recommended; a 5-7 day course is usually sufficient. In children, $H$. influenza, $S$. pneumonia, and $M$. catarrhalis are the most commonly identified organisms. A single-blinded randomized clinical trial comparing polymyxin B-trimethoprim and moxifloxacin for treatment of acute conjunctivitis in children demonstrated no difference in clinical response rate, although polymyxin B-trimethoprim was less expensive [64•]. Topical steroids should be avoided in suspected bacterial conjunctivitis.

Methicillin-resistant Staphylococcus aureus (MRSA) is an increasingly common isolate from conjunctival cultures. In a large sample of 20,180 conjunctival bacterial cultures, the percentage of MRSA rose from $7.2 \%$ in 1998 to $41.6 \%$ in 2008 [65•]. Worldwide, the proportion of MRSA in conjunctival $\mathrm{S}$. aureus isolates varies, with figures cited as high as $64 \%$ [66]. Blepharoconjunctivitis is the most common type of ocular infection by MRSA, and isolates are still highly sensitive to vancomycin [67]. Overall, conjunctival infection secondary to MRSA does not seem more severe than disease stemming from other strains of $S$. aureus. Patients with chronic systemic illness are at higher risk, likely due to their confinement to hospital wards or nursing homes where MRSA is colonized [68]. Unfortunately, the incidence of community acquired MRSA also continues to rise. In one study of a large pediatric healthcare system, $58 \%$ of ocular infections from MRSA were community acquired [69]. In this population, resistance to bacitracin and ofloxacin was high. Cultures should be taken for chronic cases and those not responding to treatment.

Severe bacterial conjunctivitis is characterized by copious purulent discharge and significant ocular inflammation. Often there is an associated exam finding of preauricular adenopathy. Culture of the discharge is warranted, and the antibiotic regimen should be targeted toward the offending bacteria once culture results are available.

Neisseria gonorrhoeae can cause hyperacute bacterial conjunctivitis in neonates and sexually active adults. Characterized by rapid onset severe purulent conjunctivitis, in untreated cases, there is high risk of corneal involvement with potential for perforation [70]. Systemic antibiotic therapy is recommended with IV ceftriaxone ( $1 \mathrm{~g}$ IM once) combined with saline lavage of the infected eye. With corneal involvement, topical treatment is also warranted. Dual therapy covering for potential concurrent chlamydial infection is recommended with either azithromycin or doxycycline. Sexual contacts should be similarly treated. Given the potential for severity of corneal disease, daily follow-up with complete anterior exam is recommended until conjunctivitis is resolved. For prevention of gonococcal ophthalmia, the US Preventive Services Task Force (USPSTD) recommends erythromycin $0.5 \%$ ophthalmic ointment within $24 \mathrm{~h}$ after delivery. Though tetracycline $1.0 \%$ is effective and a $2.5 \%$ solution of povidone-iodine may be useful in prevention of gonococcal ophthalmia neonatorum, neither are available for use in the USA [71]. Evidence suggests that due to standardized prevention strategies, blindness from gonococcal infection in the peripartum period is rare in the USA.

Adult inclusion conjunctivitis accounts for $1.8-5.6 \%$ of all acute conjunctivitis [72]. In a sexually active patient with persistent symptoms, workup for chlamydial conjunctivitis is warranted. Exam findings consistent with a chlamydial source of infection include conjunctival hyperemia, mucopurulent discharge, lymphoid follicles, chronic symptoms (over weeks to months), and concurrent genital infection. Follicles in the lower palpebral conjunctiva are the most useful identifying clinical sign and patients may display palpable preauricular adenopathy. A recent study of single dose azithromycin $(1000 \mathrm{mg})$ versus azithromycin daily (500 mg, 9 and 14 day dosing) and doxycycline (200 mg, 21 days) demonstrated no difference in treatment outcomes with regression of symptoms documented as early as 1 week. In general, 1 month was required for complete relief of symptoms [73•]. Azithromycin is available as a $1.5 \%$ ophthalmic solution, and, with a three-day treatment, is noninferior to a single dose of azithromycin in children [74]. Sexual partners of infected patients should be treated, and coinfection with chlamydia must be considered. In children with either chlamydial or gonococcal infection, child services should be informed due to the possibility of child abuse. Treatment of children with chlamydial infection is age and weight based, but infection requires systemic treatment given the potential for concurrent lung, nasopharynx, and genital tract infections [58].

\section{Allergic Conjunctivitis}

Conjunctival inflammation resulting from airborne environmental triggers such as pollens and animal dander causes the symptoms of allergic conjunctivitis. A type 1 hypersensitivity reaction in which $\operatorname{IgE}$ binds to ocular surface 
mast cells results in degranulation and release of various inflammatory mediators such as histamine, prostaglandins, and leukotrienes among others. Seasonal allergic conjunctivitis, accounting for half of all allergic conjunctivitis cases, affects a large proportion of the population and is often underdiagnosed. Symptoms typically are episodic and reappear during the spring and autumn months when exposure to allergens is highest. Typical-associated symptoms include redness, itching, tearing, watery discharge, periocular edema, and photophobia. The traditional pathognomonic symptom is intense itching, and without it, the diagnosis should be questioned [75]. Though disease is generally not sight threatening, symptoms can be severe enough to significantly impact a patients' quality of life.

Nonpharmacologic therapies are primarily used to dilute the allergens from the ocular surface and to provide relief and lubrication of the ocular surface [4]. Efforts to avoid allergen exposure should be undertaken. Since lubrication agents do little to modify the disease process, their use should be limited to mild disease or adjunctively in more advanced disease. Similarly, topical decongestants do not ameliorate the inflammatory response, and their use can be associated with rebound hyperemia. Standing use of topical decongestants is not recommended, although they can temporarily reduce chemosis and ocular redness if needed. Antihistamine agents are appropriate for mild disease, with addition of mast-cell stabilizers for persistent, recurrent or more severe symptoms; combination single drops are ideal. A brief course of topical steroids should be reserved for poorly controlled symptoms and significant inflammation despite topical antihistamine therapy. Ketorolac is also approved for treatment of allergic conjunctivitis. Fluorometholone $0.1 \%$ (FML) with olopatadine was superior to olopatadine with ketorolac $0.4 \%$ in terms of reducing redness, mucus secretion, chemosis, and eyelid edema. The two therapies were equivalent in alleviating itching, burning, and tearing [76•]. Cold compresses may enhance topical treatment with histamine antagonists [77]. A recent review found moderate evidence in support of aqueous sublingual immunotherapy to improve symptoms and reduce medications related to allergic conjunctivitis $[78 \bullet \bullet]$. Treatment by sublingual immunotherapy is prevalent in Europe and is now used off-label in the USA. Oral, in conjunction with topical pharmacotherapy, can improve ocular symptoms; and oral antihistamines are favored over leukotriene receptor antagonists [79].

Vernal conjunctivitis occurs predominantly in male children with a history of atopy and is characterized by a bilateral chronic course with many exacerbations. Symptoms acutely worsen in the spring and summer months but can gradually temper over years. Both vernal and atopic conjunctivitis can lead to significant ocular sequelae including eyelid thickening, palpebral conjunctival scarring, corneal pannus, scarring, and visual loss [58]. Classic signs of vernal conjunctivitis include giant papillary hypertrophy, limbal trantas dots, and corneal shield ulcers. General treatment should be based on severity of symptoms and begins with conservative measures including cold compresses, environmental modifications, and lubrication. Climate control with the use of air filtration may improve symptoms. Topical antihistamines and mast-cell stabilizers are typically first-line treatment if nonpharmacologic therapy is not sufficient. With severe symptoms, topical corticosteroids are often required to induce remission. Due to the relapsing pattern of disease, interest in immunomodulatory agents as steroid-sparing alternatives is growing. Studies suggest that topical cyclosporine achieves a response rapidly, but that the magnitude is small. Cyclosporine drops do not seem to completely eliminate the need for topical steroids in severe cases [80]. Tacrolimus $1 \%$ ophthalmic ointment once or twice daily demonstrated significant reduction in symptoms to $20 \%$ of baseline after 4 weeks of treatment in a small cohort [81]. For refractory giant papillae causing corneal shield ulceration, surgical resection with amniotic membrane transplantation was shown to be effective in healing and improving visual acuity in 13 eyes [82].

Atopic conjunctivitis is a chronic inflammatory condition associated with atopic dermatitis. The disease tends to present in the teen years to early twenties. Like vernal conjunctivitis, chronic uncontrolled disease can lead to permanent ocular sequelae. Boggy conjunctival edema with subepithelial fibrosis, eczematoid blepharitis with allergic shiners, and subcapsular cataract are exam findings more consistent with atopic presentation. Though symptoms can worsen seasonally, perennial disease is more common [83]. Oral antihistamines can be used in conjunction with topical medications. With refractory disease, topical and even oral steroids may be required to control symptoms. Steroids should be used judiciously secondary to side effects with longer term use [84]. A Cochrane review on topical cyclosporine for atopic keratoconjunctivitis determined that therapy provided both clinical and symptomatic relief and may reduce dependency on topical steroids. The small numbers of patients in the trials limited evaluation of safety profiles, but common adverse events were burning upon application and eyelid skin maceration [85]. Systemic immunosuppression with cyclosporine is rarely necessary. For eyelid involvement, topical tacrolimus ointment or pimecrolimus cream is effective [86•].

\section{Conclusions}

Acute conjunctivitis is a common diagnosis in both primary care and ophthalmology settings. New and emerging therapies continue to supplement the armamentarium for 
treatment of bacterial, viral, and allergic conjunctivitis. MRSA infection is increasingly common in both community and hospital acquired conjunctivitis, though microbes remain sensitive to vancomycin. Steroid-sparing agents are becoming commonplace in the treatment of conjunctivitis, whether it be for SEIs or allergic-related disease. With the continued development of accurate and rapid in-office microbe detection devices, there is greater potential for targeted therapy.

Disclosure None of the authors have reported any conflict of interests.

Human and Animal Rights and Informed Consent This article does not contain any studies with human or animal subjects performed by any of the authors.

\section{References}

Papers of particular interest, published recently, have been highlighted as:

- Of importance

•• Of major importance

1. Sheldrick JH, Wilson AD, Vernon SA, et al. Management of ophthalmic disease in general practice. Br J Gen Pract. 1993;43: 459-62.

2. Udeh BL, Schneider JE, Ohsfeldt RL. Cost effectiveness of a point-of care test for adenoviral conjunctivitis. Am J Med Sci. 2008;336:254-64.

3. Smith AF, Waycaster C. Estimate of the direct and indirect annual cost of bacterial conjunctivitis in the United States. BMC Ophthalmol. 2009;9:13.

4. Bielory BP, O'Brien TP, Bielory L. Management of seasonal allergic conjunctivitis: guide to therapy. Acta Ophthalmol. 2012;90:399-407.

5. Fitch CP, Rapoza PA, Owens S, et al. Epidemiology and diagnosis of acute conjunctivitis in an inner-city hospital. Ophthalmology. 1989;96:1215-20.

6. Isenberg SJ, Apt L, Valenton M, et al. A controlled trial of povidone-iodine to treat infectious conjunctivitis in children. Am J Ophthalmol. 2002;78:236-9.

7. Marangon FB, Millder D, Alfonso E. Laboratory results in ocular viral diseases: implications in clinical-laboratory correlation. Arq Bras Oftalmol. 2007;70:189-94.

8. Leibowitz HW, Pratt MV, Flagstad IJ, et al. Human conjunctivitis. I. Diagnostic evaluation. Arch Ophthalmol. 1976;94: 1747-9.

9. Hovding G. Acute bacterial conjunctivitis. Acta Ophthalmol. 2008;86:5-17.

10. Stenson S, Newman R, Fedukowicz H. Laboratory studies in acute conjunctivitis. Arch Ophthalmol. 1982;100:1275-7.

11. Epling J. Bacterial conjunctivitis. Clin Evid. 2010;2010:0704.

12. Infectious Agents Surveillance Center of Japan. Viruses isolated from the eye, Japan, 1990-1994. Infect Agents Surveill Rep. 1995;16:97-8.

13. Matsui K, Shimizu H, Yoshida A, et al. Monitoring of adenovirus from conjunctival scrapings in Japan during 2005-2006. J Med Virol. 2008;80:997-1003.
14. Wood SR, Sharp IR, Caul EO, et al. Rapid detection and serotyping of adenovirus by direct immunofluorescence. J Med Virol. 1997;51:198-201.

15. Rietveld RP, van Weert HC, ter Riet G, Bindels PJ. Predicting bacterial cause in infectious conjunctivitis. BMJ. 2004;329: 206-10.

16. Sambursky RP, Fram N, Cohen EJ. The prevalence of adenoviral conjunctivitis at the Wills Eye Hospital Emergency Room. Optometry. 2007;78:236-9.

17. Marangon FB, Miller D, Alfonso E. Laboratory results in ocular viral diseases: implications in clinical-laboratory correlation. Arq Bras Oftamol. 2007;70:189-94.

18. Gigliotti F, Williams WT, Hayden FG, et al. Etiology of acute conjunctivitis in children. J Pediatr. 1981;98:531-6.

19. Nauheim RC, Romanowski EG, Cruz TA, et al. Prolonged recoverability of desiccated adenovirus type 19 from various surfaces. Ophthalmology. 1990;97:1450-3.

20. Gordon YJ, Gordon RY, Romanowski EG, et al. Prolonged recovery of desiccated adenoviral serotypes 5,8, and 19 from plastic and metal surfaces in vitro. Ophthalmology. 1993;100:1835-40.

21. Kaufman HE. Adenovirus advances: new diagnostic and therapeutic options. Curr Opin Ophthalmol. 2011;22:290-3.

22. Everitt H, Wormald R, Henshaw K, et al. Viral conjunctivitis. In: Wormald R, Smeeth L, Henshaw K, editors. Evidence based ophthalmology. London: BMJ books; 2003.

23. •- Wilkins MR, Khan S, Bunce C, et al. A randomized placebocontrolled trial of topical steroid in presumed viral conjunctivitis. $\mathrm{Br}$ J Ophthalmol. 2011;95:1299-303. No significant difference found between dexamethasone and hypromellose in terms of patient discomfort or physician rating of hyperemia.

24. Trauzettel-Klosinski S, Sundmacher R, Wigand R. The effects of topical steroids in epidemic kerato-conjunctivitis. Klin Monbl Augenheilkd. 1980;176:899-906.

25. • Ward JB, Siojo LG, Waller SG. A prospective, double-masked trial of trifluridine, dexamethasone, and artificial tears for treatment of viral conjunctivitis. Ophthalmology. 2000;107:1512-7. No significant difference in outcomes of patients treated with either trifluridine, dexamethasone or artificial tears.

26. • Laibson PR, Dhiri S, Oconer J, Ortolan G. Corneal infiltrates in epidemic keratoconjunctivitis. Response to double-blind corticosteroid therapy. Arch Ophthalmol. 1970;84:36-40. Decreased apperance of subepithelial infiltrates in patients with epidemic keratoconjunctivitis when treated prophylatically with steroid eye drops when compared to nonsteroid eye drops.

27. •• Romanoski EG, Yates KA, Gordon YJ. Short-term treatment with a potent topical corticosteroid of an acute ocular adenoviral infection in the New Zealand white rabbit. Cornea. 2001;20:657-60. Treatment with 1\% prednisolone acetate significantly enhanced adenoviral replication in the rabbit ocular model when compared with the control group.

28. • Uchio E, Takeuchi S, Itoh N, et al. Clinical and epidemiological features of acute follicular conjunctivitis with special reference to that caused by herpes simplex virus type 1 . Br J Ophthalmol. 2000;84:968-72. HSV and adenoviral conjunctivitis are difficult to distinguish clinically. HSV type 1 was isolated in 4.8\% of cases of presumed epidemic keratoconjunctivitis.

29. Dosso AA, Rungger-Brandle E. Clinical course of epidemic keratoconjunctivitis: evaluation by in vivo confocal microscopy. Cornea. 2008;27:263-8.

30. Okumus S, Coskun E, Tatar MG, et al. Cyclosporine $0.05 \%$ eye drops for the treatment of subepithelial infiltrates after epidemic keratoconjunctivitis. BMC Ophthalmol. 2012;12:42.

31. Hillenkamp J, Reinhard T, Ross RS, et al. Topical treatment of acute adenoviral keratoconjunctivitis with $0.2 \%$ cidofovir and $1 \%$ cyclosporine: a controlled clinical pilot study. Arch Ophthalmol. 2001;119:1487-91. 
32. Jeng BH, Holsclaw DS. Cyclosporine A $1 \%$ eye drops for the treatment of subepithelial infiltrates after adenoviral conjunctivitis. Cornea. 2011;30:31-958.

33. Levinger E, Slomovic A, Sansanayudh W, Bahar I, Slomovic AR. Topical treatment with $1 \%$ cyclosporine for subepithelial infiltrates secondary to adenoviral keratoconjunctivitis. Cornea. 2010;29:638-40.

34. • Romanowski EG, Pless P, Yates KA, Gordon YJ. Topical cyclosporine A inhibits subepithelial immune infiltrates but also promotes viral shedding in experimental adenovirus models. Cornea. 2005;24:86-91. Topical cyclosporine A (2\% and 0.5\%) decreased formation of subepithelial infiltrates but significantly increased viral titers and duration of viral shedding in a rabbit model.

35. - Yamazaki ES, Ferraz Ca, Hazarbassonov RM, Allemann N, Campos M. Phototherapeutic keratectomy for the treatment of corneal opacities after epidemic keratoconjunctivitis. Am J Ophthalmol. 2011;151:35-43. In a prospective case series, phototherapeutic keratectomy with low dose mitomycin $C$ improved photophobia, best corrected visual acuity and contrast sensitivity in patients with subepithelial infiltrates seconary to adenovirus.

36. - Alevi D, Barsam A, Kruh J, et al. Photorefractive keratectomy with mitomycin-C for the combined treatment of myopia and subepithelial infiltrates after epidemic keratoconjunctivitis. J Cataract Refract Surg. 2012;38:1028-33. PRK with mitomycin $C$ provided good visual and refractive results in patients with subepithelial infiltrates and myopia secondary to adenovirus infection in 6 eyes.

37. Fite SW, Chodosh J. Photorefractive keratectomy for myopia in the setting of adenoviral subepithelial infiltrates. Am J Ophthalmol. 1998;126:829-31.

38. Gordon YJ, Naesens L, DeClercq E, et al. Treatment of adenoviral conjunctivitis with topical cidofovir [letter]. Cornea. 1996; $15: 546$

39. Castleton A, Kottaridis PD. A case of 'red eye' post allogeneic stem cell transplantation. Bone Marrow Transpl. 2007;39:241-2.

40. Hillenkamp J, Reinhard T, Ross RS, et al. Topical treatment of acute adenoviral keratoconjunctivitis with $0.2 \%$ cidofovir and $1 \%$ cyclosporine: a controlled clinical pilot study. Arch Ophthalmol. 2002;109:845-50.

41. Trousdale MD, Nesburn AB. Evaluation of antiherpetic activity of acyclovir in rabbits. Am J Med. 1982;73:155-60.

42. Gordon YJ, Romanowski EG, Araullo-Cru T. Topical HPMPC inhibits adenovirus type 5 in the New Zealand rabbit ocular replication model. Invest Ophthalmol Vis Sci. 1997;38:253-7.

43. Kaneko H, Mori S, Suzuki O, et al. The cotton rat model for adenovirus ocular infection: antiviral activity of cidofovir. Antiviral Res. 2004;61:63-6.

44. - Fontanelle JP, Powell CC, Veir JK, et al. Effect of topical ophthalmic application of cidofovir on experimentally induced primary ocular feline herpesvirus- 1 infection in cats. Am J Vet Res. 2008;69:289-93. Twice-daily application of 0.5\% cidofovir solution decreased viral shedding and severity of disease in cats with primary ocular feline herpesvirus-1 infection.

45. Gordon YJ, Romanoski EG, Araullo-Cruz T. Topical HPMPC inhibits adenovirus type 5 in the New Zealand rabbit ocular replication model. Invest Ophthalmol Vis Sci. 1994;35:4135-43.

46. Inoue $\mathrm{H}$, Sonoda $\mathrm{KH}$, Ishikawa $\mathrm{M}$, et al. Clinical evaluation of local ocular toxicity in candidate anti-adenoviral agents in vivo. Ophthalmologica. 2009;223:233-8.

47. Hutter H. Epidemic keratoconjunctivitis: treatment results during an epidemic. Klin Monatsbl Augenheilkd. 1991;199:192-4.

48. Pelletier JS, Stewart K, Trattler W, et al. A combination povidone-iodine $0.4 \%$ /dexamethasone $0.1 \%$ ophthalmic suspension in the treatment of adenoviral conjunctivitis. Adv Ther. 2009;26: 776-83.

49. • Clement C, Capriotti JA, Kumar M, et al. Clinical and antiviral efficacy of an ophthalmic formulation of dexamethasone povidone-iodine in a rabbit model of adenoviral keratoconjunctivitis. Invest Ophthalmol Vis Sci. 2011;52:339-44. Topical dexamethasone 0.1\%/povidone-iodine 0.4\% (FST-100) reduced clinical symptoms to a greater degree than cidofovir or Tobradex in epidemic keratoconjunctivitis infected rabbit eyes.

50. Trousdale MD, Goldschmidt PL, Nobrega R. Activity of ganciclovir against human adenovirus type-5 infection in cell culture and cotton rat eyes. Cornea. 1994;13:435-9.

51. • Yabiku ST, Yabiku MM, Bottos KM, et al. Ganciclovir $0.15 \%$ ophthalmic gel in the treatment of adenovirus keratoconjunctivitis. Arq Bras Oftamol. 2011;74:417-21. Double blind, randomized controlled trial showing non-significant trend of faster and better remission of symptoms of adenoviral conjunctivitis in patients treated with $0.15 \%$ ganciclovir gel compared to artificial tears.

52. O'Brien TP, Jeng BH, McDonald M, Raizman MB. Acute conjunctivitis: truth and misconceptions. Curr Med Res Opin. 2009;29:1953-61.

53. • Sambursky R, Trattler W, et al. Sensitivity and specificity of the AdenoPlus test for diagnosing adenoviral conjunctivitis. JAMA Ophthalmol. 2013;131:17-22. AdenoPlus showed sensitivity and specificity ranging from $85-93 \%$ and $96-99 \%$, respectively in detecting adenovirus in tear fluid.

54. Liu S, Pavan-Langston D, Colby KA. Pediatric herpes simplex of the anterior segment: characteristics, treatment and outcomes. Ophthalmology. 2012;119:2003-8.

55. Colin J, Hoh HB, Easty DL, et al. Ganciclovir ophthalmic gel (Virgan; $0.015 \%$ ) in the treatment of herpes simplex keratitis. Cornea. 1997;16:393-9.

56. • Croxtall JD. Ganciclovir ophthalmic gel $0.15 \%$ in acute herpetic keratitis (dendritic ulcers). Drugs. 2011;71:603-10. Randomized, phase III trial in patients with acute herpetic keratitis showing effective clinical resolution of dendritic ulcers with ganciclovir ophthalmic gel $0.15 \%$ following 7 days of treatment.

57. Wilhelmus KR. Antiviral treatment and other therapeutic interventions for herpes simplex virus and epithelial keratitis. Cochrane Database Syst Rev. 2010;8(12):CD002898. doi: 10.1002/ 14651858.CD002898.pub4.

58. American Academy of Ophthalmology. Cornea/external disease panel. Preferred practice pattern guidelines: conjunctivitis. San Francisco: American Academy of Ophthalmology; 2013.

59. Colby K, Pavan-Langston D. Author reply. Ophthalmology. 2013;120:e28-9.

60. Puri LR, Shrestha GB, Shah DN, Chaudhary M, Thakur A. Ocular manifestations in herpes zoster ophthalmicus. Nepal J Ophthalmol. 2011;3:165-71.

61. Huff JC, Bean B, Balfour HH Jr, et al. Therapy of herpes zoster with oral acyclovir. Am J Med. 1988;85:84-9.

62. Rietveld RP, van Weert HC, ter Riet G, Bindels PJ. Diagnostic impact of signs and symptoms in acute infectious conjunctivitis: systemic literature search. BMJ. 2003;327:789.

63. Sheikh A, Hurwitz B. Antibiotics versus placebo for acute bacterial conjunctivitis. Cochrane Database Syst Rev. 2006;19(2): CD001211. doi: 10.1002/14651858.CD001211.pub2.

64. - Williams L, Malhotra Y, Murante B, Laverty S, et al. A singleblinded randomized clinical trial comparing polymyxin B-trimethoprim and moxifloxacin for treatment of acute conjunctivitis in children. J Pediatr. 2013;162:857-61. Randomized trial demonstrating equal efficacy of polymyxin B-trimethoprim and moxifoxacin hydrochloride for treatment of acute conjunctivitis with decreased cost of polymyxin B-trimethoprim. 
65. - Adebayo A, Parikh JG, McCormick SA. Shifting trends in in vitro antibiotic susceptibilities for common bacterial conjunctival isolates in the last decade at the New York Eye and Ear Infirmary. Graefes Arch Clin Exp Ophthalmol. 2011;249:111-9. Review demonstrating a significant increase in percentrage of MRSA isolated in conjunctival bacterial cultures from 1997-2008.

66. Tazawa H, Ooisi M. MRSA ocular infections. Nippon Rinsho. 1992;50:1122-6.

67. Freidlin J, Acharya N, Lietman TM, et al. Spectrum of eye disease caused by methicillin-resistant staphylococcus aureus. Am J Ophthalmol. 2007;144:313-5.

68. Shanmuganathan VA, Armstrong $M$, Buller A, Tullo AB. External ocular infections due to methicillin-resistant Staphylococcus aureus (MRSA). Eye (Lond). 2005;19:284-91.

69. Amato M, Pershing S, Walvick M, Tanaka S. Trends in ophthalmic manifestations of methicillin-resistant Staphylococcus aureus (MRSA) in a northern California pediatric population. J AAPOS. 2013;17:243-7.

70. Kawashima M, Kawakita T, Den S, Tomita M, Shimazaki J. Surgical management of corneal perforation secondary to gonococcal keratoconjunctivitis. Eye (Lond). 2009;23:339-44.

71. Preventative US. Services Task Force. Ocular prophylaxis for gonococcal ophthalmia neonatorum: reaffirmation recommendation statement. Am Fam Physician. 2012;85:197-8.

72. Barney N, Azari A. Conjunctivitis: a systemic review of diagnosis and treatment. JAMA. 2013;310:1721-9.

73. - Malamos P, Georgalas I, Rallis K, et al. Evaluation of singledose azithromycin versus standard azithromycin/doxycycline treatment and clinical assessment of regression course in patients with adult inclusion conjunctivitis. Curr Eye Res. 2013;38: 1198-206. Single dose azithromycin demonstrates equivalent retreatment rate when compared to long term azithromycin and doxycylcine regimens in patients with adult inclusion conjunctivitis.

74. Garnock-Jones KP. Azithromycin 1.5\% ophthalmic solution: in purulent bacterial or trachomatous conjunctivitis. Drugs. 2012;72:361-73.

75. Bielory L, Friedlaender M. Allergic conjunctivitis. Immunol Allergy Clin North Am. 2008;28:58-73.

76. - Celik T, Turkoglu EB. Comparative evaluation of olopatadine $0.01 \%$ combined fluorometholone $0.1 \%$ treatment versus olopatadine $0.01 \%$ combined ketorolac $0.4 \%$ treatment in patients with acute seasonal allergic conjunctivitis. Curr Eye Res.
2014;39:42-6. Ranomized, placebo-controlled multicenter study. Fluorometholone was better than ketorolac (when used comitantly with olapatadine) in relieving redness, chemosis, mucous secretion and eyelid edema in patients with seasonal allergic conjunctivitis.

77. Bilkhu PS, Wolffsohn JS, Naroo SA, Robertson L, Kennedy R. Effectiveness of nonpharmacologic treatments for acute seasonal allergic conjunctivitis. Ophthalmology. 2014;121(1):72-8.

78. • Lin SY, Erekosima N, Kim JK, et al. Sublingual immunotherapy for the treatment of allergic rhinoconjunctivitis and asthma: A systemic review. JAMA. 2013;309:1278-88. Moderate grade level of evidence supporting sublingual immunotherapy for treatment of allergic rhinoconjunctivitis and asthma.

79. Gane J, Buckley RJ. Leukotriene receptor antagonists in allergic eye disease: A systemic review and meta-analysis. J Allergy Clin Immunol Pract. 2013;1:64-74.

80. Vichyanond P, Kosrirukvongs P. Use of cyclosporine A and tacrolimus in treatment of vernal keratoconjunctivitis. Curr Allergy Asthma Rep. 2013;3:308-14.

81. Vichyanond P, Tantimongkolsuk C, Dumrongkigchaiporn P, et al. Vernal keratoconjunctivitis: Results of a novel therapy with $0.1 \%$ topical ophthalmic FH-506 ointment. J Allergy Clin Immunol. 2004;113:355-8.

82. Guo P, Kheirkhah A, Zhou WW, Qin L, Shen XL. Surgical resection and amniotic membrane transplantation for treatment of refractory giant papillae in vernal keratoconjunctivitis. Cornea. 2013;32:816-20.

83. Chen JJ, Applebaum DS, Sun GS, Pflugfelder SC. Atopic keratoconjunctivitis: a review. J Am Acad Dermatol. 2014;70(3): $569-575$.

84. Guglielmetti S, Dart JK, Calder V. Atopic keratoconjunctivitis and atopic dermatitis. Curr Opin Allergy Clin Immunol. 2010;10: 478-85.

85. Gonzalez-Lopez JJ, Lopez-Alcalde J, Morcillo Laiz R, Fernandez Buenaga R, Rebolleda Fernandez G. Topical cyclosporine for atopic keratoconjunctivitis. Cochrane Database Syst Rev. 2012;9(2):CD009078. doi: 10.1002/14651858.CD0009078.pub2.

86. • Ohashi Y, Ebihara N, Fujishima H, et al. A randomized, placebo controlled trial of tacrolimus ophthalmic suspension $0.1 \%$ in severe allergic conjunctivitis. J Ocul Pharmacol Ther. 2010;26: 164-74. Randomized, double-masked, placebo-controlled trial demonstrating that tacrolimus ophthalmic suspension (0.1\%) was effective in reducing giant papillae and corneal involvement from severe allergic conjunctivitis. 